\title{
THE ADO METHOD FOR SOLVING ONE-DIMENSIONAL DEEP-PENETRATION TRANSPORT PROBLEMS
}

\author{
D. L. Ribeiro ${ }^{a}$, \\ M. P. Rodrigues ${ }^{b}$, \\ and J. F. Prolo Filho ${ }^{\text {a }}$ \\ ${ }^{a}$ Universidade Federal do Rio Grande \\ Programa de Pós-Graduação em Engenharia Oceânica \\ Av. Itália, km 8 \\ CEP 96203-900, Rio Grande, RS, Brasil \\ lopesribeirodalvana20@gmail.com \\ joaoprolo@furg.br \\ ${ }^{\mathrm{b}}$ Universidade Federal de Pelotas \\ Programa de Pós-Graduação em Ciência e Engenharia \\ dos Materiais \\ R. Gomes Carneiro, 1 - Centro, \\ CEP 96010-610, Pelotas, RS, Brasil \\ marco.paulsen.rodrigues@gmail.com \\ Received: February 28, 2019 \\ Revised: March 21, 2019 \\ Accepted: April 22, 2019

\section{ABSTRACT} \\ In this work, the Analytical Discrete Ordinate method (ADO method) is \\ used to solve deep-penetration transport problems in one-dimensional \\ Cartesian geometry, subject to isotropic and linear anisotropic scattering \\ effects. The regime is considered permanent, the media are homogeneous, \\ and the fluxes are caused by sources located on the boundaries of the \\ domain. In addition, the energy fluctuations will be considered as not \\ significant, characterizing the phenomena as monoenergetic problems. In \\ order to validate the code, method and provide benchmark results, some \\ test problems will be treated and results will be discussed. In particular, \\ the ADO method generated fairly accurate results when compared to \\ other methods based on $\mathrm{S}_{\mathrm{N}}$ approaches, at a relatively low computational \\ cost. \\ Keywords: deep-penetration transport problem; homogeneous medium; \\ isotropic scattering; anisotropic scattering
}

\section{NOMENCLATURE}

A,D eigenvalue problem matrices

$\mathrm{A}_{\mathrm{j}} \quad$ homogeneous solution coefficient

$\mathrm{N} \quad \mathrm{S}_{\mathrm{N}}$ quadrature order

$\mathrm{R} \quad$ reflectance coefficient

$\mathrm{T}$ transmittance coefficient

$\mathrm{U}, \mathrm{V}$ auxiliary functions

$\mathrm{w}_{\mathrm{k}} \quad$ quadrature weights

$\mathrm{x}$ spatial variable, $\mathrm{m}$

\section{Greek symbols}

$\lambda_{j} \quad$ eigenvalues of the homogeneous problem

$\mu \quad$ directional variable

$\mu_{\mathrm{k}} \quad$ quadrature points

$v_{j} \quad$ separation constants

$\Phi \quad$ elementary solutions

$\phi \quad$ scalar flux on $x, \mathrm{n} / \mathrm{m}^{2}$ s

$\Psi \quad$ angular flux on $x$ and $\mu, \mathrm{n} / \mathrm{cm}^{2}$.s

$\sigma_{\mathrm{s} 0} \quad$ isotropic macroscopic cross section, $\mathrm{cm}^{-1}$

$\sigma_{\mathrm{s} 1} \quad$ linearly anisotropic cross section, $\mathrm{cm}^{-1}$

$\sigma_{\mathrm{t}}$ total macroscopic cross section, $\mathrm{cm}^{-1}$

\section{Subscripts}

$\mathrm{i}, \mathrm{k}$ directions indexes

j separation constant index

\section{INTRODUCTION}

Deep-penetration problems, characterized by domains with several mean free paths (mfp) of extension, correspond to one of the most important class of problems in particle transport area, mainly due to their applicability on the processes involved in nuclear reactors. In particular, the potential application is in radiological protection issues, more precisely on shielding calculations (Oliveira, 2007), very important for personnel, equipment and the environment protection.

In this sense, the mathematical modeling of the involved physical phenomena and the development of more efficient methods for their treatment are important points that allow a better analysis and predicting processes, at a low operational cost.

There are several methods in the literature which approach may follow a probabilistic or deterministic way to solve these problems, but they usually have limitations as to the geometry complexity or precision method, therefore increasing the processing costs, independently of computing capacity and parallel processing resources.

Among the deterministic methods (considered most suitable for the treatment of deep-penetration problems (Yang et. al., 2018)), the Analytical Discrete Ordinates Method (ADO method) is presented here. Formulations based on the ADO method (Barichello and Siewert, 1999) have been intensively and 
successfully used for solving, in an accurate and concise way, a wide range of Rarefied Gas Dynamics problems (Scherer et al., 2009), transport problems in one-dimensional media (Prolo Filho and Rodrigues, 2017) and in different geometries (Barichello et al., 2002; Ferreira et al., 2015). Besides providing closed form solutions for the discrete-ordinates version of the integro-differential transport equation (analytical in terms of the spatial variable), the independence of iterative schemes and computational meshes can be mentioned. The reduced size of the associated eigenvalues problem in comparison to other methodologies inspired by the $S_{N}$ approach, also make this formulation more efficient in the computational point of view.

Thus, the ADO method will be used here to study a class of neutron transport problems in nonmultiplicative media, in one-dimensional Cartesian geometry, under permanent regime, that suffer isotropic and linear anisotropic scattering effects, subject to constant sources located at the domain boundaries. In particular, phenomena that involve some anisotropic degree are directly related to reactorcore reactions (Poveshchenko, 2016).

According to ADO method, from the integrodifferential transport equation, a discrete-ordinate version is generated using the half-range GaussLegendre quadrature set (Sykes, 1951; Barichello and Siewert, 1999; Prolo Filho and Ribeiro, 2018). For the resultant ordinary differential equations system, an expression for the neutron angular fluxes in terms of decreasing exponentials is proposed. In the process, elementary solutions and separation constants are obtained through a reduced eigenvalue problem. The expressions for the angular neutron fluxes, analytical in terms of the spatial variable, are written. In the end, boundary conditions imposed for the problems are used to determine certain coefficients of the general solution.

This way, the next sections are dedicated to presenting the discrete-ordinates solution based on ADO method for some test cases that can be related to deep-penetration problems, as well as discussing the physical parameters effect on the interest quantities. Some benchmark results are presented, and new ones are provided.

\section{MATHEMATICAL MODEL}

According Barichello (1992), the monoenergetic neutron transport equation in permanent regime for a one-dimensional Cartesian geometry, applied to a homogeneous medium with linearly anisotropic scattering, is written as

$$
\begin{aligned}
& \mu \frac{d}{d x} \Psi(x, \mu)+\sigma_{t} \Psi(x, \mu)= \\
& \quad \frac{\sigma_{s 0}}{2} \int_{-1}^{1} \Psi\left(x, \mu^{\prime}\right) d \mu^{\prime}+\frac{3}{2} \sigma_{s 1} \mu \int_{-1}^{1} \mu^{\prime} \Psi\left(x, \mu^{\prime}\right) d \mu^{\prime}
\end{aligned}
$$

with $x[\mathrm{~cm}]$ and $\mu$ corresponding, respectively, to the spatial and directional variables wherein the angular fluxes $\Psi(x, \mu)\left[\mathrm{n} / \mathrm{cm}^{2}\right.$ s $]$ are evaluated, $\sigma_{t}\left[\mathrm{~cm}^{-1}\right], \sigma_{s 0}$ $\left[\mathrm{cm}^{-1}\right]$ and $\sigma_{s 1}\left[\mathrm{~cm}^{-1}\right]$ are the total, the isotropic and the linearly anisotropic macroscopic cross sections. Here, there is an interest in dealing with transport problems without inner sources, Fig. 1, so that the fluxes are caused by prescribed non-homogeneous boundary conditions and particular solutions are not necessary.

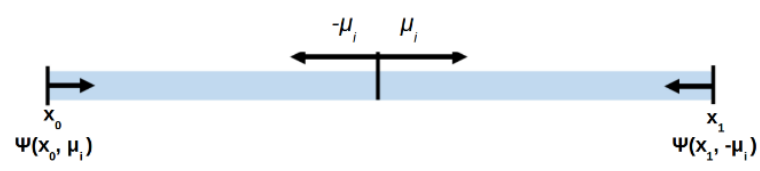

Figure 1. Domain of the homogeneous neutron transport problems.

In order to apply the ADO method, a quadrature scheme is applied, making it possible to write a discrete ordinates version of Eq. (1), such as

$$
\begin{aligned}
& \mu_{i} \frac{d}{d x} \Psi\left(x, \mu_{i}\right)+\sigma_{t} \Psi\left(x, \mu_{i}\right)= \\
& \quad \frac{\sigma_{s 0}}{2} \sum_{k=1}^{N} w_{k}\left[\Psi\left(x, \mu_{k}\right)+\Psi\left(x,-\mu_{k}\right)\right]+ \\
& \quad \frac{3}{2} \sigma_{s 1} \mu_{i} \sum_{k=1}^{N} w_{k} \mu_{k}\left[\Psi\left(x, \mu_{k}\right)-\Psi\left(x,-\mu_{k}\right)\right], \\
& -\mu_{i} \frac{d}{d x} \Psi\left(x,-\mu_{i}\right)+\sigma_{t} \Psi\left(x,-\mu_{i}\right)= \\
& \frac{\sigma_{s 0}}{2} \sum_{k=1}^{N} w_{k}\left[\Psi\left(x, \mu_{k}\right)+\Psi\left(x,-\mu_{k}\right)\right]- \\
& \quad \frac{3}{2} \sigma_{s 1} \mu_{i} \sum_{k=1}^{N} w_{k} \mu_{k}\left[\Psi\left(x, \mu_{k}\right)-\Psi\left(x,-\mu_{k}\right)\right]
\end{aligned}
$$

where $i=1, \ldots, N$, being $N$ the number of discrete directions of the Gauss-Legendre quadrature set (Stroud and Secrest, 1966) mapped within [0, 1] interval, with quadrature points $\mu_{k}$ associated with weights $w_{k}$. Moreover, $\mu_{i}$ represents the positive directions, while $-\mu_{i}$ represents the negative ones.

Is important to highlight that, despite the versatility of the ADO method for quadratures, the use of a half-range scheme provides better results than full-range Gauss-Legendre (Bell and Glasstone, 1979), which causes a poor representation of the angular fluxes near the contours, especially when $\mu$ is closer to zero, or under free surface boundary conditions. To facilitate the distinction between the different formulations presented here, the ADO method associated with half-range quadrature will be indicated as $\mathrm{ADO}_{2 \mathrm{~N}}$.

\section{THE ADO METHOD}

Following some basic steps of the ADO method (Barichello and Siewert, 1999), a homogeneous solution for the transport problem described by Eqs. (2)-(3) is proposed in the form 


$$
\Psi\left(x, \pm \mu_{i}\right)=\Phi\left(v, \pm \mu_{i}\right) e^{-x / v}
$$

for $i=1, \ldots, N$, where the separation constant $v$ is associated with elementary solutions $\Phi\left(\nu, \pm \mu_{i}\right)$.

This way, substituting Eq. (4) into Eqs. (2)-(3), the coupled algebraic system is obtained

$$
\begin{aligned}
& -\frac{\mu_{i}}{v} \Phi\left(v, \mu_{i}\right)+\sigma_{t} \Phi\left(v, \mu_{i}\right)= \\
& \quad \frac{\sigma_{s 0}}{2} \sum_{k=1}^{N} w_{k}\left[\Phi\left(v, \mu_{k}\right)+\Phi\left(v,-\mu_{k}\right)\right]+ \\
& \quad \frac{3}{2} \sigma_{s 1} \mu_{i} \sum_{k=1}^{N} w_{k} \mu_{k}\left[\Phi\left(v, \mu_{k}\right)-\Phi\left(v,-\mu_{k}\right)\right] \\
& \frac{\mu_{i}}{v} \Phi\left(v,-\mu_{i}\right)+\sigma_{t} \Phi\left(v,-\mu_{i}\right)= \\
& \frac{\sigma_{s 0}}{2} \sum_{k=1}^{N} w_{k}\left[\Phi\left(v, \mu_{k}\right)+\Phi\left(v,-\mu_{k}\right)\right]- \\
& \quad \frac{3}{2} \sigma_{s 1} \mu_{i} \sum_{k=1}^{N} w_{k} \mu_{k}\left[\Phi\left(v, \mu_{k}\right)-\Phi\left(v,-\mu_{k}\right)\right]
\end{aligned}
$$

for $i=1, \ldots, N$.

Now, two auxiliary functions are defined as

$$
\begin{aligned}
& U\left(v, \mu_{i}\right)=\Phi\left(v, \mu_{i}\right)+\Phi\left(v,-\mu_{i}\right), \\
& V\left(v, \mu_{i}\right)=\Phi\left(v, \mu_{i}\right)+\Phi\left(v,-\mu_{i}\right),
\end{aligned}
$$

such that, if Eqs. (5)-(6) are added, the expression

$$
V\left(v, \mu_{i}\right)=\frac{v}{\mu_{i}}\left[\sigma_{t} U\left(v, \mu_{i}\right)-\sigma_{s 0} \sum_{k=1}^{N} w_{k} U\left(v, \mu_{k}\right)\right]
$$

is obtained. On the other hand, subtracting Eq. (6) from Eq. (5), another relation between $U\left(v, \mu_{i}\right)$ and $V\left(\nu, \mu_{i}\right)$ is given by

$$
\begin{aligned}
& \frac{\mu_{i}}{v} U\left(v, \mu_{i}\right)+\sigma_{t} V\left(v, \mu_{i}\right)= \\
& \quad 3 \sigma_{s 1} \mu_{i} \sum_{k=1}^{N} w_{k} \mu_{k} V\left(v, \mu_{k}\right) .
\end{aligned}
$$

From Eqs. (9)-(10), after some manipulations, an eigenvalue problem in terms of $U\left(\nu, \mu_{i}\right)$ is obtained, in the form

$$
\begin{array}{r}
\frac{1}{v^{2}} U\left(v, \mu_{i}\right)=\frac{\sigma_{t}^{2}}{\mu_{i}^{2}} U\left(v, \mu_{i}\right)-\sum_{k=1}^{N} w_{k} U\left(v, \mu_{k}\right) \times \\
{\left[\frac{\sigma_{t} \sigma_{s 0}}{\mu_{i}^{2}}+3 \sigma_{t} \sigma_{s 1}-3 \sigma_{s 0} \sigma_{s 1}\left(\sum_{j=1}^{N} w_{j}\right)\right],}
\end{array}
$$

for $i=1, \ldots, N$. The matrix representation of Eq. (11) is given by

$$
[D-A] U=\lambda U,
$$

where $\boldsymbol{U}$ is a vector with components $U\left(\nu, \mu_{i}\right)$,

$$
\lambda=\frac{1}{v^{2}},
$$

and the matrices of dimension $N \times N$ for Eq. (12) are such that

$$
D=\operatorname{diag}\left[\left(\frac{\sigma_{t}}{\mu_{1}}\right)^{2},\left(\frac{\sigma_{t}}{\mu_{2}}\right)^{2}, \ldots,\left(\frac{\sigma_{t}}{\mu_{N}}\right)^{2}\right]
$$

and

$$
A(i, k)=w_{k}\left[\frac{\sigma_{t} \sigma_{s 0}}{\mu_{i}^{2}}+3 \sigma_{t} \sigma_{s 1}-3 \sigma_{s 0} \sigma_{s 1}\left(\sum_{j=1}^{N} w_{j}\right)\right]
$$

for $i, k=1, \ldots, N$.

With the eigenvalues problem solved, the values of $\lambda_{j}$ for $j=1, \ldots, N$ are used to calculate the separation constants $v_{j}$ by Eq. (13) and, from Eqs. (7)-(8), the elementary solutions can be written as

$$
\Phi\left(v_{j}, \mu_{i}\right)=\frac{1}{2}\left[U\left(v_{j}, \mu_{i}\right)+V\left(v_{j}, \mu_{i}\right)\right],
$$

$$
\Phi\left(v_{j},-\mu_{i}\right)=\frac{1}{2}\left[U\left(v_{j}, \mu_{i}\right)-V\left(v_{j}, \mu_{i}\right)\right],
$$

for $i, j=1, \ldots, N$.

Since the separation constants occur in pairs, $\left\{ \pm v_{j}\right\}$, with real values, the symmetry is imposed in order to build a linearly independent basis of elementary solutions. For that

$$
\begin{aligned}
& \Phi\left(v_{j}, \mu_{i}\right)=\Phi\left(-v_{j},-\mu_{i}\right), \\
& \Phi\left(v_{j},-\mu_{i}\right)=\Phi\left(-v_{j}, \mu_{i}\right),
\end{aligned}
$$

for $i, j=1, \ldots, N$.

Thus, the solution for Eqs. (2)-(3), in an explicit form, is given by

$$
\begin{gathered}
\Psi\left(x, \mu_{i}\right)=\sum_{j=1}^{N} A_{j} \Phi\left(v_{j}, \mu_{i}\right) e^{-\left(x-x_{0}\right) / v_{j}}+ \\
A_{j+N} \Phi\left(-v_{j}, \mu_{i}\right) e^{-\left(x_{1}-x\right) / v_{j}}, \\
\Psi\left(x,-\mu_{i}\right)=\sum_{j=1}^{N} A_{j} \Phi\left(v_{j},-\mu_{i}\right) e^{-\left(x-x_{0}\right) / v_{j}}+ \\
A_{j+N} \Phi\left(-v_{j},-\mu_{i}\right) e^{-\left(x_{1}-x\right) / v_{j}}
\end{gathered}
$$

for $i=1, \ldots, N$.

Here, the arbitrary constants $A_{j}$ are to be determined, and they depend on the conditions imposed for the problems.

It is important to observe that, in this 
formulation, from a set of $2 N$ discrete ordinates equations, an eigenvalue problem of order $N$ was derived, which means a relevant gain in comparison to other similar discrete ordinates approaches, where characteristic equations or eigensystems of order $2 \mathrm{~N}$ are obtained, for a similar quadrature scheme (Vilhena and Barichello, 1991; Nunes and Barros, 2009).

\section{COUPLING SYSTEM}

Despite the $\mathrm{ADO}_{2 \mathrm{~N}}$ method having proven to be effective in dealing with more general boundary conditions, here it will be focused on problems with prescribed boundary conditions. So, in order to explicitly define the solution for a class of problems proposed here, the following

$$
\begin{gathered}
\Psi\left(x_{0}, \mu_{i}\right)=F, \\
\Psi\left(x_{1},-\mu_{i}\right)=G,
\end{gathered}
$$

will be used for $i=1, \ldots, N$, being $F$ and $G$ constant incident fluxes.

Equations (20)-(23) lead to a $2 N \times 2 N$ system which solution provides the value of all coefficients $A_{j}$ and, consequently, makes Eqs. (20)-(21) fully established, which makes it possible to analyze some quantities of interest.

\section{NUMERICAL RESULTS AND COMPUTATIONAL ASPECTS}

For the method presented here, some test cases are described on Tab. 1 , where the $\mathrm{ADO}_{2 \mathrm{~N}}$ method is compared with other analytical and numerical methods for validation.
In order to generate some numerical results, quantities of interest were chosen, such as:

a) Scalar flux or total flux (Bell and Glasstone, 1979), given by

$$
\phi(x)=\int_{-1}^{1} \Psi\left(x, \mu^{\prime}\right) d \mu^{\prime},
$$

corresponding to the mean of the angular flux in terms of the directional variable;

b) Reflectance or reflection coefficient where, following Clements and Özisik (1983), is defined as

$$
R=\frac{\int_{0}^{1} \mu^{\prime} \Psi\left(x_{0},-\mu^{\prime}\right) d \mu^{\prime}}{\int_{0}^{1} \mu^{\prime} \Psi\left(x_{0}, \mu^{\prime}\right) d \mu^{\prime}}
$$

that makes the relation between the incident and reflected radiation on a surface at $x=x_{0}$;

c) Transmittance or transmission coefficient (Clements and Özisik, 1983), which measures the fraction of incident radiation that is transmitted through the medium, established at $x=x_{1}$, as

\begin{tabular}{|c|c|c|c|c|c|c|c|c|}
\hline Problem & $\begin{array}{l}\text { Quantity of } \\
\text { Interest }\end{array}$ & Characteristics & $\sigma_{t}$ & $\sigma_{\mathrm{s} 0}$ & $\sigma_{s 1}$ & $F$ & $G$ & {$\left[x_{0}, x_{1}\right]$} \\
\hline 1 & $\begin{array}{l}\text { Scalar } \\
\text { Flux }\end{array}$ & $\begin{array}{l}\text { Homogeneous } \\
\text { Isotropic }\end{array}$ & 1.00 & 0.97 & 0.00 & 2.00 & 2.00 & {$[0.0,50.0]$} \\
\hline 2 & $\begin{array}{l}\text { Scalar } \\
\text { Flux }\end{array}$ & $\begin{array}{l}\text { Homogeneous } \\
\text { Anisotropic }\end{array}$ & 1.00 & 0.99 & 0.80 & 1.00 & 0.00 & {$[0.0,100.0]$} \\
\hline 3 & $\begin{array}{c}\text { Reflectance } \\
\text { Transmittan } \\
\text { ce }\end{array}$ & $\begin{array}{l}\text { Homogeneous } \\
\text { Isotropic }\end{array}$ & 1.00 & $\begin{array}{l}\text { Select } \\
\text { cases }\end{array}$ & 0.00 & 1.00 & 0.00 & $\begin{array}{l}\text { Select } \\
\text { Cases }\end{array}$ \\
\hline 4 & $\begin{array}{l}\text { Scalar } \\
\text { Flux }\end{array}$ & $\begin{array}{l}\text { Homogeneous } \\
\text { Anisotropic }\end{array}$ & 1.00 & 0.50 & $\begin{array}{l}\text { Select } \\
\text { Cases }\end{array}$ & 1.00 & 0.00 & {$[0.0,10.0]$} \\
\hline
\end{tabular}

$$
T=\frac{\int_{0}^{1} \mu^{\prime} \Psi\left(x_{1},-\mu^{\prime}\right) d \mu^{\prime}}{\int_{0}^{1} \mu^{\prime} \Psi\left(x_{0}, \mu^{\prime}\right) d \mu^{\prime}}
$$

Table 1. Physical parameters used for each test case in this work.

In Nunes and Barros (2009), Problem 1 was solved by using three different numerical methods: Diamond Difference (DD), Step and CN method. All of them, besides using the discrete ordinate version of the transport equation, subdivide the domain into cells, where average angular fluxes are established. The numeric aspects consist in how the relationship between neighboring cells is, and an iterative process is used to compute them.

The $\mathrm{ADO}_{2 \mathrm{~N}}$ method, on the other hand, does not need iterative methods or interpolation processes and, when applied to homogeneous media, does not require the domain to be split into cells. The expressions for the solutions are analytical in terms of the spatial variable, making it simple to obtain the scalar fluxes in any spatial position of interest.

In terms of agreement (Tab. 2) the $\mathrm{ADO}_{2 \mathrm{~N}}$ method becomes closer to DD, getting almost all significant digits tabled. The $\mathrm{ADO}_{2 \mathrm{~N}}$ results presented on Tab. 2 with $N=4$ are convergent and the resulting symmetry agrees with the proposed parameters and phenomenon. 
It is emphasized here that convergence is faster in problems involving only isotropic effects, and it is not necessary to use higher quadrature orders to obtain good results.

Table 2. Problem 1 - Convergence analysis and results validation for Scalar flux profiles.

\begin{tabular}{c|c|ccc}
\hline \hline & $\mathrm{ADO}_{2 \mathrm{~N}}$ & DD & Step & $\mathrm{CN}$ \\
$x$ & $N=4$ & $N=8$ & $N=8$ & $N=8$ \\
\hline 0.0 & 1.704731 & 1.704731 & 1.703435 & 1.704683 \\
5.0 & 0.366806 & - & - & - \\
10.0 & 0.083341 & - & - & - \\
15.0 & 0.018983 & - & - & - \\
20.0 & 0.004523 & - & - & - \\
25.0 & 0.001954 & 0.001956 & 0.002012 & 0.001958 \\
30.0 & 0.004523 & - & - & - \\
35.0 & 0.018983 & - & - & - \\
40.0 & 0.083341 & - & - & - \\
45.0 & 0.366806 & - & - & - \\
50.0 & 1.704731 & 1.704731 & 1.703435 & 1.704683 \\
\hline \hline
\end{tabular}

In Barichello (1992), results for Problem 2 (Tab. 3) were generated by $\mathrm{LTS}_{\mathrm{N}}$ method, which consists in an approach based on Laplace Transform, and profiles were compared with $\mathrm{SGF}_{\mathrm{N}}$ method (Barros and Larsen, 1990).

The $\mathrm{LTS}_{\mathrm{N}}$ formulation is a semi-analytical method (as $\mathrm{ADO}_{2 \mathrm{~N}}$ method) where, after the discrete ordinates approximation of the Eq. (1), an algebraic system is generated by the application of Laplace Transform on $x$ variable, making it necessary to deal with imaginary values. The $\mathrm{SGF}_{\mathrm{N}}$ method works like the numerical methods reported on Problem 1 . However, the source terms are treated analytically.

The formulation presented here, besides the good features as the analyticity of the solutions, does not use complex variable techniques, once all parameters that arise during the process are real.

Regarding the agreement of the results (Tab. 3) all methods show similar values, presenting differences, eventually, in the last two digits. The shape of the profiles agrees with the proposed parameters and the deep-penetration phenomenon, in the sense of the scalar flux decay as it moves away from the source.

This same problem was recently studied by Barbosa (2018), who used a version of ADO method associated with full-range Gauss-Legendre quadrature (which will be referred here as $\mathrm{ADO}_{\mathrm{N}}$ ), and also compared their results with those obtained by $\mathrm{LTS}_{\mathrm{N}}$ and $\mathrm{SGF}_{\mathrm{N}}$. There, using $N=8$, the same agreement with the literature in terms of significant digits was obtained and, only with $N=64$ it was able to reproduce the values obtained here by $\mathrm{ADO}_{2 \mathrm{~N}}$ with $N$ $=8$ (this work). Thus, it can be said that the results for $\mathrm{ADO}_{2 \mathrm{~N}}$ with $N=8$ are convergent and reliable.

Table 3. Problem 2 - Convergence analysis and results validation for Scalar flux profiles computed by $\mathrm{ADO}_{2 \mathrm{~N}}$ method and the literature (Barichello, 1992; Barros and Larsen, 1990).

\begin{tabular}{c|ccc|cc}
\hline \hline & & & $\mathrm{ADO}_{2 N}$ & $\mathrm{LTS}_{N}$ & $\mathrm{SFG}_{N}$ \\
$x$ & $N=4$ & $N=6$ & $N=8$ & $N=8$ & $N=8$ \\
\hline 0.0 & 0.82299 & 0.82299 & 0.82299 & 0.82284 & 0.82284 \\
10.0 & 0.36023 & 0.36023 & 0.36023 & - & - \\
20.0 & 0.16653 & 0.16654 & 0.16654 & - & - \\
30.0 & $0.76992 \times 10^{-1}$ & $0.76993 \times 10^{-1}$ & $0.76993 \times 10^{-1}$ & - & - \\
40.0 & $0.35593 \times 10^{-1}$ & $0.35593 \times 10^{-1}$ & $0.35593 \times 10^{-1}$ & - & - \\
50.0 & $0.16452 \times 10^{-1}$ & $0.16452 \times 10^{-1}$ & $0.16452 \times 10^{-1}$ & $0.16471 \times 10^{-1}$ & $0.16470 \times 10^{-1}$ \\
60.0 & $0.75988 \times 10^{-2}$ & $0.75989 \times 10^{-2}$ & $0.75989 \times 10^{-2}$ & - & - \\
70.0 & $0.34977 \times 10^{-2}$ & $0.34977 \times 10^{-2}$ & $0.34977 \times 10^{-2}$ & - & - \\
80.0 & $0.15838 \times 10^{-2}$ & $0.15838 \times 10^{-2}$ & $0.15838 \times 10^{-2}$ & - & - \\
90.0 & $0.66037 \times 10^{-3}$ & $0.66037 \times 10^{-3}$ & $0.66037 \times 10^{-3}$ & - & - \\
100.0 & $0.12221 \times 10^{-3}$ & $0.12222 \times 10^{-3}$ & $0.12222 \times 10^{-3}$ & $0.12251 \times 10^{-3}$ & $0.12250 \times 10^{-3}$ \\
\hline \hline
\end{tabular}

Results for Problem 3 were generated here and compared to Schulz (2014). The difference between both formulations lies on the treatment of the spatial variable in the dimensionless form, $\tau=\sigma_{t} x$. However, considering a medium with $\sigma_{t}=1.0$, both are equivalent ( $x$ and $\tau$ ) and comparisons can be performed without any difficulties.

During the simulations, a serie of values for reflectance and transmittance were obtained, and some could be verified within the literature. A very good agreement was obtained, sometimes differing in the last significant digit, depending on the length of the domain or the isotropic scattering coefficient.
Table 4. Problem 3 - Reflection coefficient computed by $\mathrm{ADO}_{2 \mathrm{~N}}$ method (this work) using $N=50$.

\begin{tabular}{cccc}
\hline$x_{1}$ & $\sigma_{\mathrm{s} 0}=0.20$ & $\sigma_{\mathrm{s} 0}=0.50$ & $\sigma_{\mathrm{s} 0}=0.99$ \\
\hline 0.04 & 0.00685247 & 0.01757597 & 0.03634189 \\
0.05 & 0.00831499 & 0.02143109 & 0.04469452 \\
0.07 & 0.01101701 & 0.02865066 & 0.06072115 \\
0.10 & 0.01460163 & 0.03843267 & 0.08329296 \\
0.33 & 0.03121851 & 0.08768257 & 0.21809644 \\
0.45 & 0.03573454 & 0.10271706 & 0.27102243 \\
0.49 & 0.03688701 & 0.10673589 & 0.28683488 \\
0.50 & 0.03715297 & 0.10767608 & 0.29066267 \\
1.00 & 0.04393448 & 0.13416516 & 0.43596159 \\
2.00 & 0.04607244 & 0.14508538 & 0.58800003 \\
5.00 & 0.04626466 & 0.14654094 & 0.73750699 \\
\hline \hline
\end{tabular}


In the analysis of results on Tables 4-5, it was possible to observe that both reflectance and transmittance maintains a direct relation with the isotropic scattering parameter, so that higher values of $R$ and $T$ are obtained for greater values of $\sigma_{s}$.

Table 5. Problem 3 - Transmission coefficient computed by $\mathrm{ADO}_{2 \mathrm{~N}}$ method (this work) using $N=50$.

\begin{tabular}{ccll}
\hline \hline$x_{1}$ & $\sigma_{s 0}=0.20$ & $\sigma_{s 0}=0.50$ & $\sigma_{s 0}=0.99$ \\
\hline 0.04 & 0.93347421 & 0.94415819 & 0.96285882 \\
0.05 & 0.91810777 & 0.93115585 & 0.95430656 \\
0.07 & 0.88858229 & 0.90606388 & 0.93788086 \\
0.10 & 0.84695598 & 0.87043785 & 0.91471092 \\
0.33 & 0.60110334 & 0.65304658 & 0.77533943 \\
0.45 & 0.50803024 & 0.56686834 & 0.72004243 \\
0.49 & 0.48085205 & 0.54119699 & 0.70344155 \\
0.50 & 0.47432335 & 0.53499287 & 0.69941681 \\
1.00 & 0.24626874 & 0.30670882 & 0.54435238 \\
2.00 & 0.07274150 & 0.10705530 & 0.37334749 \\
5.00 & 0.00244613 & 0.00528758 & 0.17315224 \\
\hline \hline
\end{tabular}

On the other hand, this behavior does not last in relation to domain length since the increase of $x_{1}$ leads to higher values of $R$, but reduces the value of T. Moreover, as can be seen in Figs. 2-3, these behaviors are not linear and reflectance tends to stabilize for longer domains.

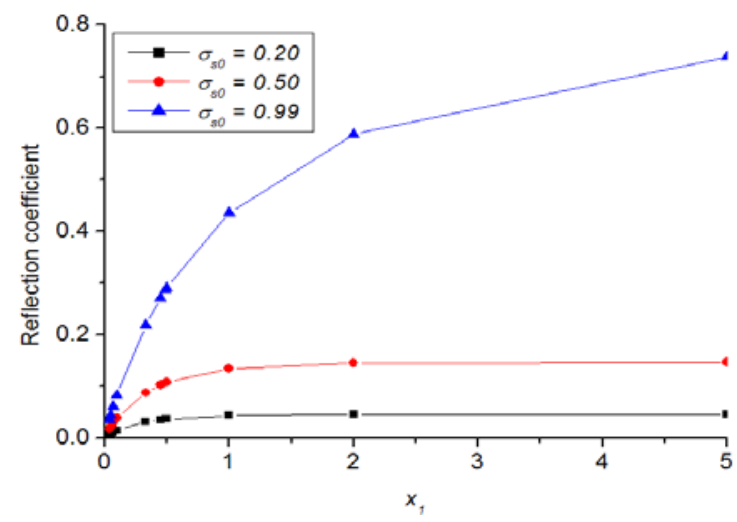

Figure 2. Problem 3 - Reflection coefficients computed by $\mathrm{ADO}_{2 \mathrm{~N}}$ based on Tab. 4 .

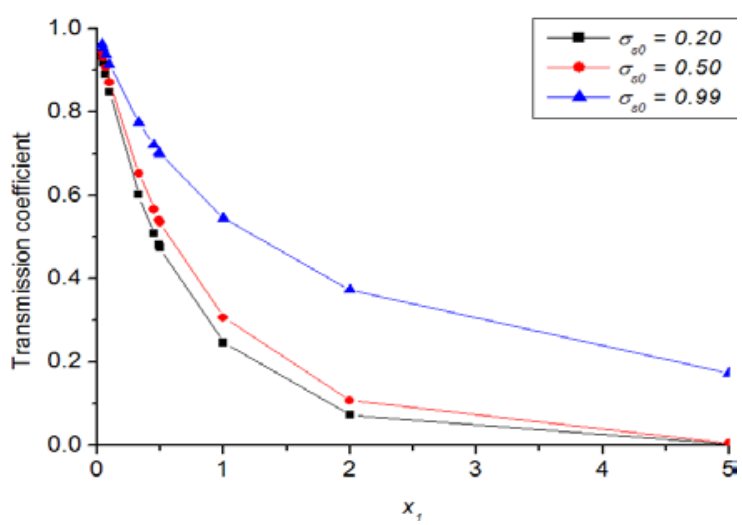

Figure 3. Problem 3 - Transmission coefficients computed by $\mathrm{ADO}_{2 \mathrm{~N}}$ based on Tab. 5 .
In the results of Problem 4 (Tab. 6 and Fig. 4), the effects of the anisotropy factor on the scalar flux profiles are observed. First, independently of $\sigma_{s 1}$ value, it is verified that the profiles maintain the expected exponential decay for the deep-penetration phenomenon.

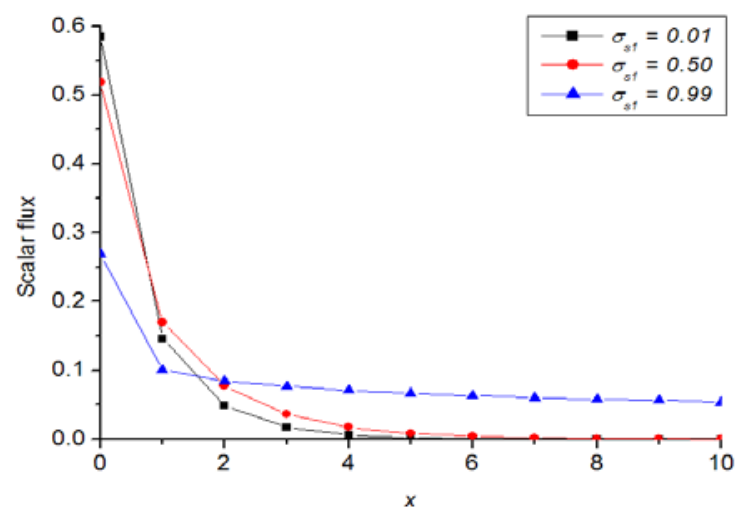

Figure 4. Problem 4 - Scalar flux profiles computed by $\mathrm{ADO}_{2 \mathrm{~N}}$ based on Tab. 6 .

Table 6. Problem 4 - Scalar flux profiles computed by $\mathrm{ADO}_{2 \mathrm{~N}}$ method (this work) using $N=20$.

\begin{tabular}{cccc}
\hline \hline$x$ & $\sigma_{s 1}=0.01$ & $\sigma_{s 1}=0.50$ & $\sigma_{s 1}=0.99$ \\
\hline 0.0 & 0.5848157 & 0.5190318 & 0.2688959 \\
1.0 & 0.1456423 & 0.1699366 & 0.1004884 \\
2.0 & 0.0490466 & 0.0773185 & 0.0844114 \\
3.0 & 0.0174581 & 0.0367612 & 0.0768457 \\
4.0 & 0.0063741 & 0.0177487 & 0.0713892 \\
5.0 & 0.0023611 & 0.0086275 & 0.0669800 \\
6.0 & 0.0008826 & 0.0042084 & 0.0633603 \\
7.0 & 0.0003319 & 0.0020574 & 0.0604427 \\
8.0 & 0.0001252 & 0.0010087 & 0.0581929 \\
9.0 & 0.0000468 & 0.0004982 & 0.0566082 \\
10.0 & 0.0000144 & 0.0002379 & 0.0535436 \\
\hline \hline
\end{tabular}

In addition, profiles with higher anisotropic scattering coefficients have lower initial values (closer to the source) and lower decay along the domain, providing the graphic a flattened shape.

\section{CONCLUSIONS}

The present work showed the viability and performance of the ADO method on the solution of typical deep-penetration neutron transport problems, in one-dimensional Cartesian geometry, where it was possible to compare some results with the available literature and provide some benchmark profiles. On the tables presented in this study, convergence of the results is noted when changing the quadrature order. The increasing number of quadrature points leads to better representation of the integral term, despite also increasing the number of discrete directions. In particular, it could be observed in Problem 2 that the use of a half-range quadrature scheme contributes to accelerating the convergence of the results, even for media with linear anisotropy effects. In general, the 
profiles presented a good agreement with the literature, approximately three to five significant digits, depending on the problem's class and the involved parameters.

During the simulations, it was observed that the anisotropy factor of the medium causes convergence difficulties, but more accurate results can be obtained by simply increasing the number of Gauss-Legendre quadrature points. Here, some good features of the $\mathrm{ADO}_{2 \mathrm{~N}}$ method can be highlighted, hence no iterative schemes were used and the computational effort was relatively low, spending less than 2 seconds (in a 3.10 $\mathrm{GHz}$ Intel Core I7 processor with 8GB of RAM) for each profile. Part of this performance was due to the reduced order eigenvalue system and the explicit form of the solutions, analytical in terms of the spatial variable. The code, which implementation is simple, was developed making use of the free software Octave 4.2.1, using a half-range Gauss Legendre quadrature set, working with any quadrature order. Furthermore, all profiles showed compatible physical behavior in terms of the parameters, proposal of solutions and boundary conditions used.

For future works, the authors intend to vary the anisotropy degree and change the geometry configuration of the problem in order to represent transport phenomena in heterogeneous layered media.

\section{ACKNOWLEDGEMENTS}

The authors would like to thank FAPERGS for research financial support.

\section{REFERENCES}

Barbosa, B. V., 2018, Desenvolvimento de uma Interface Gráfica para o Tratamento de Problemas de Transporte de Nêutrons, Universidade Federal do Rio Grande - FURG, Rio Grande, RS. (in Portuguese)

Barichello, L. B., 1992, Formulação Analítica para Solução do Problema de Ordenadas Discretas Unidimensional, Doctoral Thesis, PROMEC-UFRGS, Porto Alegre, RS. (in Portuguese)

Barichello, L. B., and Siewert, C. E., 1999, A Discrete-Ordinates Solution for a Non-Grey Model with Complete Frequency Redistribution, Journal of Quantitative Spectroscopy and Radiative Transfer, Vol. 62, No. 6, pp. 665-675.

Barichello, L. B., Rodrigues, P., and Siewert, C. E., 2002, An Analytical Discrete-Ordinates Solution for Dual-mode Heat Transfer in a Cylinder, Journal of Quantitative Spectroscopy and Radiative Transfer, Vol. 73, No. 6, pp. 583-602.

Barros, R. C., and Larsen, E. W., 1990, A Numerical Method for One-Group Slab-Geometry Discrete Ordinates Problems with no Spatial Truncation Error, Nuclear Science and Engineering, Vol. 104, No. 3, pp. 199-208.

Bell, G. I., and Glasstone, S., 1979, Nuclear Reactor Theory, Krieger Publishing Co. Inc.

Clements, T. B., and Özisik, M. N., 1983,
Effects of Stepwise Variation of Albedo on Reflectivity and Transmissivity of an Isotropically Scattering Slab, International Journal of Heat and Mass Transfer, Vol. 26, No. 10, pp. 1419-1426.

Ferreira, C. E. S., Emmendorfer, L. R., and Prolo Filho, J. F., 2015, Formulação Nodal Aplicada à Problemas de Transporte Bidimensional em Geometria Cartesiana, Scientia Plena, Vol. 11, No. 8, pp. 081315 1-10. (in Portuguese)

Nunes, C. E. A., and Barros, R. C., 2009, Aplicativo Computacional para Cálculos de Blindagem com Modelo de Transporte $\mathrm{S}_{\mathrm{N}}$ Unidimensional e Monoenergético, in: International Nuclear Atlantic Conference, Rio de Janeiro, pp. 1-13. (in Portuguese)

Oliveira, F. B. S., 2007, Problema Inverso de Reconstrução Analítica Aproximada da Solução da Equação de Transporte de Partículas Neutras Monoenergéticas em Geometria Unidimensional Cartesiana com Espalhamento Isotrópico, Doctoral Thesis, IPRJ-UERJ, Nova Friburgo, RJ. (in Portuguese)

Poveshchenko, T. S., 2016, Computational Analysis of a Nuclear Reactor Cell in the LinearAnisotropic Approximation by the Generalized Method of First Collisions Probabilities, Atomic Energy, Vol. 120, No. 2, pp. 95-99.

Prolo Filho, J. F., and Rodrigues, M. P., 2017, Application of the ADO Method on DisadvantageFactor Calculation for Heterogeneous Slab Cells Considering Linear Anisotropic Scattering Effects, Scientia Plena, Vol. 13, No. 1, pp. 049905 1-10.

Prolo Filho, J. F., and Ribeiro, D. L., 2018, Evaluation of the Half-Range Quadrature Scheme for the Treatment of One-Dimensional Transport Problems in Homogeneous Media, in: $8^{a}$ Conferência Sul em Modelagem Computacional, Universidade Federal do Rio Grande, Rio Grande, pp. 1369-1381.

Scherer, C. S., Prolo Filho, J. F., and Barichello, L. B., 2009, An Analytical Approach to the Unified Solution of Kinetic Equations in Rarefied Gas Dynamics. I. Flow Problems, Zeitschrift für Angewandte Mathematik und Physik, Vol. 60, No. 1, pp. 70-115.

Schulz, D. M., 2014, Métodos Analíticos e Computacionais em Geofísica Nuclear, PPGMApUFRGS, Porto Alegre, RS. (in Portuguese)

Stroud, A. H., and Secrest, D., 1966, Gaussian Quadrature Formulas, Prentice Hall Inc.

Sykes, J. B., 1951, Approximate Integration of the Equation of Transfer, Monthly Notices of the Royal Astronomical Society, Vol. 111, pp. 377-386.

Vilhena, M. T., and Barichello, L. B., 1991, A New Analytical Approach to Solve the Neutron Transport Equation, Kerntechnik, Vol. 56, No. 5, pp. 334-336.

Yang, W., Yuan, B., Zhang, S., Guo, H., Liu, Y., and Deng, L., 2018, A Neutron Transport Calculation Method for Deep-Penetration and its Preliminary Verification, in: 26th International Conference on Nuclear Engineering, London, pp. V003T02A032 1-6. 